\title{
Correction to: S(+)-ketamine
}

\section{Current trends in emergency and intensive caremedicine}

\author{
H. Trimmel · R. Helbok · T. Staudinger · W. Jaksch · B. Messerer · H. Schöchl · R. Likar
}

Published online: 29 June 2018

(C) The Author(s) 2018

\section{Correction to:}

Wien Klin Wochenschr 2017

https://doi.org/10.1007/s00508-017-1299-3

The article $\mathrm{S}(+)$-ketamine Current trends in emergency and intensive caremedicine, written by Helmut Trimmel, Raimund Helbok, Thomas Staudinger, Wolfgang Jaksch, Brigitte Messerer, Herbert Schöchl and Rudolf Likar, was originally published electronically on the publisher's internet portal (currently SpringerLink) on 10 January 2018 without open access. With the author(s)' decision to opt for Open Choice the copyright of the article changed on 13 June 2018 to (C) The Author(s) 2018 and the article is forthwith distributed under the terms of the Creative Commons Attribution 4.0 International License (http:// creativecommons.org/licenses/by/4.0/), which permits use, duplication, adaptation, distribution and reproduction in any medium or format, as long as you give appropriate credit to the original author(s) and the source, provide a link to the Creative Commons license and indicate if changes were made.
The online version of the original article can be found under https://doi.org/10.1007/s00508-017-1299-3.

Prim. Dr. H. Trimmel, MSc ( $₫)$

Department of Anaesthesia, EmergencyMedicine and Intensive Care and Karl Landsteiner Institute of Emergency Medicine, General Hospital Wiener Neustadt, Corvinusring 3-5, 2700 Wiener Neustadt, Austria

Helmut.Trimmel@wienerneustadt.lknoe.at

PD OA Dr. R. Helbok

University Hospital for Neurology, Medical University

Innsbruck, Innsbruck, Austria

raimund.helbok@i-med.ac.at

Prof. Dr. T. Staudinger

Department of Medicine I, Medical University of Vienna,

Vienna, Austria

Thomas.Staudinger@meduniwien.ac.at
OA Dr. W. Jaksch

Department for Anaesthesia, Intensive Care and Pain Medicine, Wilhelminen Hospital of the City of Vienna,

Vienna, Austria

Wolfgang.Jaksch@wienkav.at

OÄ Dr. B. Messerer

Department for Cardiothoracic Anaesthesia, Medical

University of Graz, Graz, Austria

Brigitte.Messerer@medunigraz.at

PD OA Dr. H. Schöchl

AUVA Trauma Hospital, Salzburg, Austria

Herbert.Schoechl@auva.at

Prim. Prof. Dr. R. Likar, MSc

Department for Anaesthesia and Intensive Care, General

Hospital of Klagenfurt, Klagenfurt, Austria

Rudolf.Likar@kabeg.at 